Apidologie, 1978, 9 (4), 273-290.

\title{
SUR QUELQUES FACTEURS INFLUENÇANT \\ LE DÉVELOPPEMENT DE MEGACHILE PACIFICA PANZ (HYMENOPTERA, MEGACHILIDAE)
}

\author{
Über den Einfluss einiger Faktoren auf die Entwicklung \\ von Megachile pacifica Panz (Hymenoptera, Megachilidae)
}

\author{
J. N. TASEI et M. M. MASURE
}

Laboratoire de Zoologie, I.N.R.A., S.A.P.F. 86000 Lusignan

\author{
SUMMARY \\ FACTORS INFLUENCING THE DEVELOPMENT \\ OF MEGACHILE PACIFICA PANZ (HYMENOPTERA, MEGACHILIDAE)
}

The authors, working in controlled conditions studied the influence of several factors on the development rate of the megachile immature stages.

The period of larval development is longer when the larvae belong to the first generation; when they are going to diapause or when they are fed with only one pollen (Melilotus) in comparison with a two pollen bee bread (Melilotus + Medicago sativa). The French and Canadian strains have similar development rates that is : $18-19$ days at $22^{\circ} \mathrm{C}$ and $10-11$ days at $29^{\circ} \mathrm{C}$. The temperature $15^{\circ} \mathrm{C}$ kills all eggs and young larvae, and does not prevent old larvae from pupating.

The length of the hibernation storage and the incubation temperature applied on hibernated prepupae are the main factors responsible for the emergence pattern. Long hibernation storage at $5-7^{\circ} \mathrm{C}$ correspond to short incubation periods within the range 1 to 8 months of cold. A hibernation period longer than 8-10 months results in a higher diapause intensity. Temperature requirements for emergence are lower when a cool temperature $\left(17^{\circ} \mathrm{C}\right)$ is applied during the feeding stage. Alternating incubation temperatures : $29^{\circ} \mathrm{C}-15^{\circ} \mathrm{C}$ are more favourable to post-diapause development than the constant temperature $22^{\circ} \mathrm{C}$. The French strain has higher temperature requirements than the Canadian.

\section{RESUMÉ}

Les auteurs ont étudié en conditions contrôlées l'influence de plusieurs facteurs sur la vitesse de développement des stades immatures des mégachiles.

Le développement est plus lent lorsque les larves appartiennent à la première génération; lorsqu'elles sont destinées à entrer en diapause, ou lorsqu'elles s'alimentent sur un seul pollen (Melilotus) plutôt que sur 
deux (Melilotus + Medicago sativa). Les souches françaises et canadiennes ont des vitesses de développement identiques : $18-19$ jours à $22^{\circ} \mathrm{C}$ et $10-11$ jours à $29^{\circ} \mathrm{C}$. La température $15^{\circ} \mathrm{C}$ tue les œufs et les jeunes larves et n'empêche pas la nymphose.

La longueur de l'hibernation et la température d'incubation appliquée aux prénymphes sortant d'hibernation, sont les principaux facteurs influant sur les délais d'émergence. A des hibernations longues à 5 $7^{\circ} \mathrm{C}$, correspondent des incubations courtes, du moins lorsque la période de froid varie de 1 à 8 mois. Une hibernation durant plus de 8-10 mois renforce l'intensité de la diapause. Les besoins en chaleur nécessaires à l'émergence diminuent lorsque l'on applique pendant la période d'alimentation une température basse $\left(17^{\circ} \mathrm{C}\right)$. L'alternance des températures d'incubation $\left(29^{\circ} \mathrm{C}-15^{\circ} \mathrm{C}\right)$ est plus favorable au développement des larves sortant d'hibernation, que la température constante $22^{\circ} \mathrm{C}$. La souche française a des besoins en chaleur supérieurs à ceux de la souche canadienne.

\section{INTRODUCTION}

Les techniques d'élevage de Megachile pacifica pollinisateur de la luzerne, se sont perfectionnées depuis les premières années de la domestication de cette espèce vers 1960 aux États-Unis. Ces améliorations découlent pour la plupart de travaux de recherches ayant trait au comportement de nidification, à la biologie des ennemis naturels, à la physiologie du développement. Ce dernier domaine est particulièrement important puisque l'on élève des mégachiles de souche américaine dans les deux hémisphères sous des latitudes très diverses, du $37^{\mathrm{e}}$ au $58^{\mathrm{e}}$ parallèle si l'on considère l'Amérique du Nord. Les conditions de photopériode et de thermopériode auxquelles sont soumises les insectes sont donc très variées. Par ailleurs, il faut rappeler que le bon déroulement de l'hibernation et de l'incubation, phases du cycle annuel qui ont lieu à des températures constantes, est une des conditions du succès de la phase estivale d'activité des adultes.

Signalons les principales acquisitions dans ce domaine : on connait les réactions des adultes et des larves à certaines températures très élevées ou très basses, de même que la gamme des températures favorables à l'hibernation. Par contre il n'y a aucune étude complète sur certaines questions importantes telles que celle du voltinisme; seuls quelques éléments dans la littérature, parfois contradictoires, sont la preuve de la complexité du déterminisme de l'arrêt de développement larvaire des mégachiles. Aucune explication globale de l'induction de la diapause n'est donnée.

Il nous est apparu qu'une meilleure connaissance des facteurs pouvant agir sur le développement est un travail fondamental préliminaire devant précéder toute étude du déterminisme de l'arrêt du développement. Nous faisons ici une analyse des causes de variations de la vitesse de développement chez les stades immatures aussi bien pendant la phase estivale de ponte précédant l'hibernation que pendant la phase post-hivernale lors de la reprise du développement devant conduire à la nymphose des larves et à l'émergence des adultes de la première génération.

\section{MATÉRIEL ET MÉTHODES}

Afin de ne pas faire $\mathbf{r}$. ier plus d'un facteur par expérience nous avons travaillé sur des élevages indépendants en compartiments vitrés de serre abritant une ou deux espèces florales cultivées en caissettes. 
D'autre part, les cellules construites par les femelles sont prélevées soit le jour de la ponte, soit avant l'éclosion de l'œuf, puis elles sont isolées dans de petites boites en polystyrène (de $20 \times 20 \times 12 \mathrm{~mm}$ ). Enfin elles sont soumises à différents traitements thermiques, l'humidité des enceintes étant toujours maintenue aux environs de $60 \%$ d'H.R.

La Figure 1 permet de situer dans le temps et de caractériser les différentes périodes du cycle annuel des mégachiles de souche américaine.

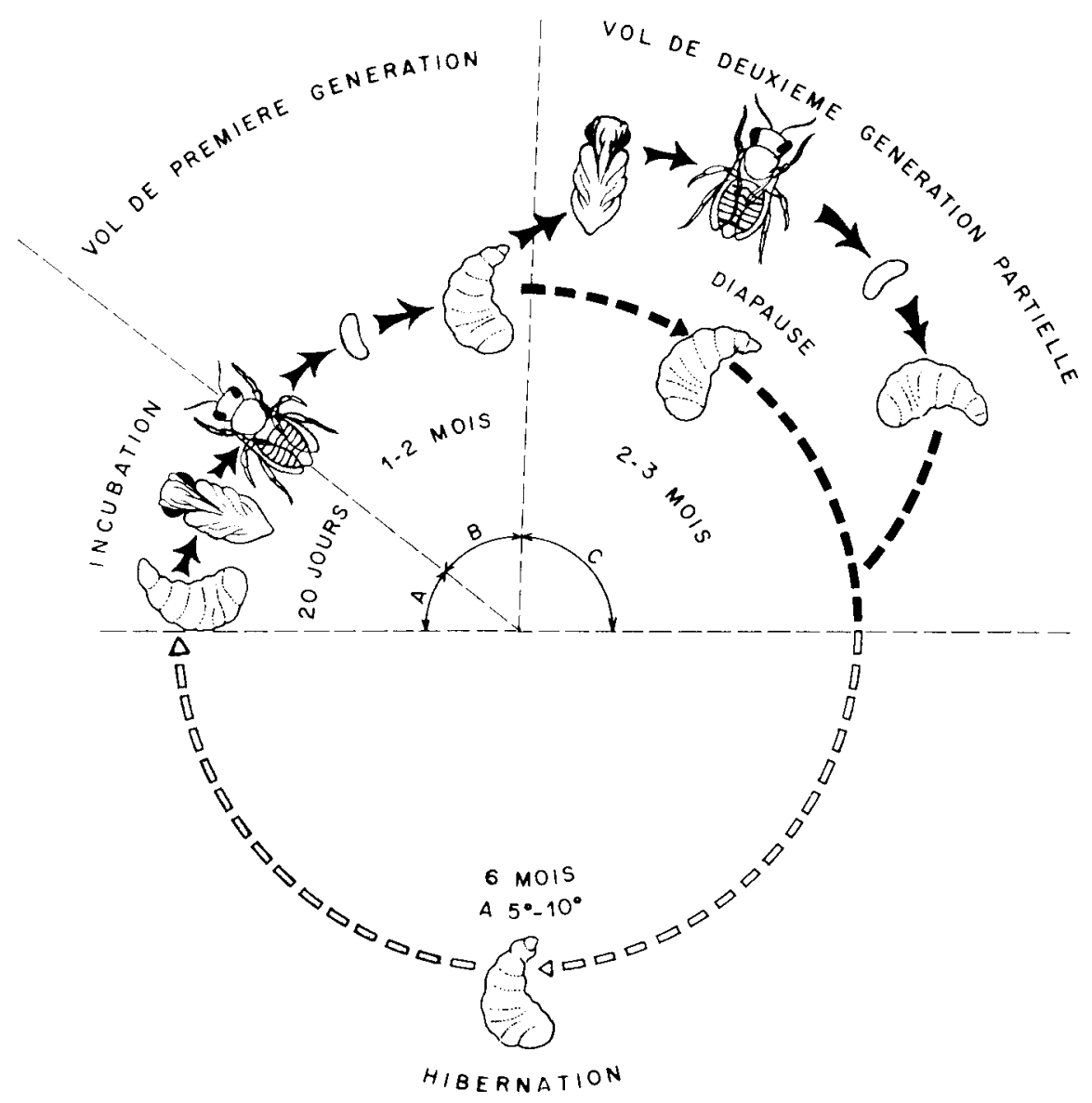

Fig. 1. - Cycle annuel de Megachile pacifica américain élevé en France.

$\square \square \square=$ phase hivernale.

$\longrightarrow$ - phase estivale.

$\mathrm{A}=$ réactivation par incubation $\dot{\mathrm{a}} 29^{\circ} \mathrm{C}$.

$\mathrm{B}=$ vol de $\mathrm{I}^{\mathrm{re}}$ génération.

$\mathrm{C}=$ vol de $2^{\mathrm{e}}$ génération partielle et entrée en diapause larvaire partielle.

Aвв. 1. - Jahreszyklus von Megachile pacifica aus Amerika, aufgezogen in Frankreich.

Winterphase

$\longrightarrow$ Sommerphase

$\mathrm{A}=$ Aktivierung durch Inkubation bei $29^{\circ} \mathrm{C}$.

$\mathrm{B}=$ Flug der ersten Generation.

$\mathrm{C}=$ Flug eines Teiles der zweiten Generation, die teilweise im Larvenstadium in Diapause getreten war. 
Pendant la phase estivale au cours du vol de première et deuxième génération on mesure les longueurs des différentes périodes du développement en fonction de la température, du sexe, de la nourriture, de l'origine géographique, de l'état de diapause, et du rang de la génération (premier ou second vol).

Pendant la phase post-hivernale on mesure les délais d'incubation nécessaires à l'obtention de l'image selon l'origine géographique, le traitement thermique subi avant l'entrée en diapause ou appliqué durant l'hibernation. Lors de certaines expériences on est amené à utiliser des insectes issus d'élevages de masse en champ. Chaque fois que c'est le cas on le mentionne au début de l'exposé des résultats.

\section{RÉSULTATS}

A. Vitesse du développement pendant la phase estivale

1) Influence de la température sur le développement (fig. 2)

- A $15^{\circ} \mathrm{C}$ constants tous les oufs et les jeunes larves meurent. Les larves âgées seules peuvent évoluer et malgré cette basse température elles arrivent à se nymphoser.

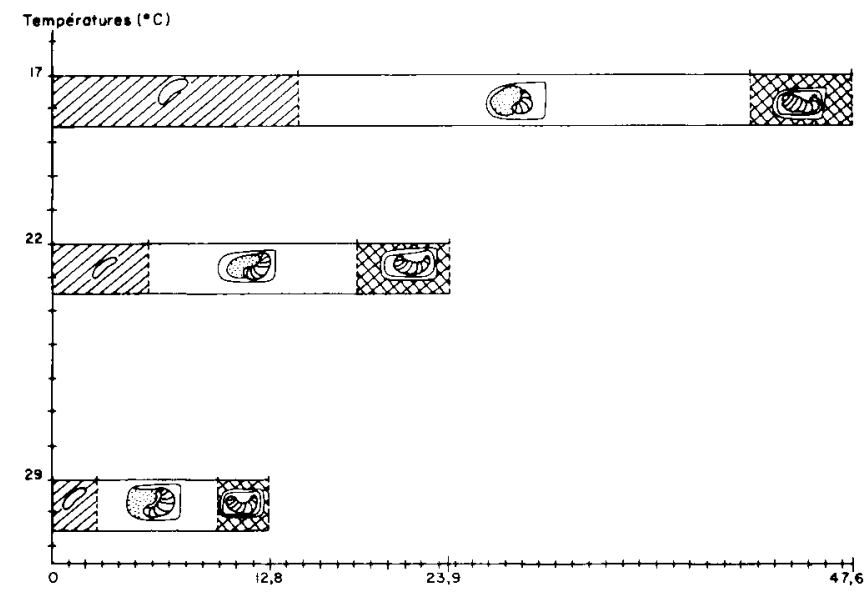

FIG. 2. - Vitesse de développement à 3 températures constantes.

Verticalement $=$ températures.

Horizontalement $=$ délais de développement en jour.

$\square \backslash$ = incubation de l'œuf.

$\square=$ stade d'alimentation.

$\bar{X}=$ filage du cocon.

Aвв. 2. - Entwicklungsgeschwindigkeit bei drei konstanten Temperaturen.

Vertikal = Temperaturen.

Horizontal = Entwicklungsverzögerung (in Tagen).

$\nabla J=$ Inkubation des Eis.

$\square=$ Frassstadium.

$\square=$ Spinnen des Kokons. 
- A $17^{\circ} \mathrm{C}$ constants la mortalité dépasse $20 \%$ des individus et affecte surtout les larves. Le développement dure 47 jours (de l'œuf à la fin du filage du cocon par la larve âgée).

- A $22^{\circ} \mathrm{C}$ ce temps de développement est réduit à 24 jours et à $29^{\circ} \mathrm{C}$ à 13 jours.

\section{2) Influence de la nourriture (tableau 1)}

Les larves de souche américaine destinées à entrer en diapause ont une période de développement sensiblement plus courte (17 jours) lorsqu'elles se nourrissent sur une pâtée pollinique mixte (luzerne + melilot) alors que sur une provision uniflorale (melilot) le développement se déroule en près de 19 jours. Le filage du cocon n'est pas affecté par la modification du régime, seule la période pendant laquelle la larve s'alimente et mue varie selon la composition de la nourriture.

TABL. 1. - Nourriture et vitesse du développement postembryonnaire

à $22^{\circ} \mathrm{C}$ de Megachile pacifica de souche canadienne.

TAB. 1. - Ernährung und postembryonale Entwicklungsgeschwindigkeit bei $22^{\circ} \mathrm{C}$ von Megachile pacifica Stamm Kanada.

\begin{tabular}{|c|c|c|c|c|}
\hline \multirow{2}{*}{$\begin{array}{c}\text { Nombre } \\
\text { d'individus (1) } \\
\text { Zahl der Individuen }\end{array}$} & \multirow[b]{2}{*}{$\begin{array}{l}\text { Plantes butinées } \\
\text { Besuchte Pflanzen }\end{array}$} & \multicolumn{3}{|c|}{$\begin{array}{l}\text { Durée en jours } \\
\text { Dauer (in Tagen) }\end{array}$} \\
\hline & & $\begin{array}{c}\text { Stade } \\
\text { d'alimentation } \\
\text { Frassstadium }\end{array}$ & $\begin{array}{l}\text { Filage du cocon } \\
\text { Spinnen des Kokons }\end{array}$ & $\begin{array}{c}\text { Développement } \\
\text { larvaire total } \\
\text { Gesamte } \\
\text { Larvenentwicklung }\end{array}$ \\
\hline 30 & Melilotus alba & 12,7 & 6 & 18,7 \\
\hline \multirow[t]{2}{*}{19} & $\begin{array}{c}\text { Melilotus alba } \\
+ \\
\text { Medicago sativa }\end{array}$ & 11,2 & 5,9 & 17,1 \\
\hline & & $* t n=3,05^{* *}(2)$ & " $t » N . S$. & $\alpha t n=2,62^{* *}$ \\
\hline
\end{tabular}

(1) Tous les individus appartiennent à la première génération annuelle et entrent en diapause après le filage du cocon.

(2) * : * $\mathrm{t}$ » significatif à $1 \%$.

(1) Alle Individuen gehören zur ersten Generation des Jahres und treten nach dem Spinnen des Kokons in die Diapause.

(2) ** : « $t$ » signifikant bei $1 \%$.

\section{3) Influence de l'état de "préparation de la diapause" (tableau 2)}

Les larves qui sont destinées à entrer en diapause après le filage du cocon sont plus lentes à se développer ( 18 jours à $22^{\circ} \mathrm{C}$ et 10 jours à $29^{\circ} \mathrm{C}$ ) que celles qui sont destinées à se nymphoser pour donner une deuxième génération annuelle (15 jours à $22^{\circ} \mathrm{C}$ et 9 jours à $29^{\circ} \mathrm{C}$ ). 
TABt. 2. - Préparation de la diapause et vitesse du développement postembryonnaire a $22{ }^{\circ} \mathrm{C}$ et $29^{\circ} \mathrm{C}$ de Megachile pacifica de souche canadienne.

TAB. 2. - Vorbereitung der Diapause und postembryonale Entwicklungsgeschwindigkeit bei $22^{\circ} \mathrm{C}$ und bei $29^{\circ} \mathrm{C}$ von Megachile pacifica, Stamm Kanada.

\begin{tabular}{|c|c|c|c|c|c|}
\hline \multirow{2}{*}{$\begin{array}{l}\text { Température } \\
\text { Temperatur }\end{array}$} & \multirow{2}{*}{$\begin{array}{c}\text { Nombre } \\
\text { d'individus } \\
\text { Anzahl } \\
\text { der Individuen }\end{array}$} & \multirow{2}{*}{$\begin{array}{c}\text { Induction } \\
\text { de la diapause } \\
\text { Induktion } \\
\text { der Diapause }\end{array}$} & \multicolumn{3}{|c|}{$\begin{array}{l}\text { Durée en jours } \\
\text { Dauer (in Tagen) }\end{array}$} \\
\hline & & & $\begin{array}{c}\text { Stade } \\
\text { d'alimentation } \\
\text { Frassstadium }\end{array}$ & $\begin{array}{c}\text { Filage } \\
\text { du cocon } \\
\text { Spinnen } \\
\text { des Kokons }\end{array}$ & $\begin{array}{c}\text { Développement } \\
\text { larvaire total } \\
\text { Gesamte } \\
\text { Larvenentwicklung }\end{array}$ \\
\hline \multirow[t]{2}{*}{$22^{\circ} \mathrm{C}$} & \multirow[t]{2}{*}{$\begin{array}{r}120 \\
17\end{array}$} & \multirow[t]{2}{*}{$\begin{array}{l}+ \\
0\end{array}$} & $\begin{array}{l}12,5 \\
11,9\end{array}$ & $\begin{array}{l}5,7 \\
3,5\end{array}$ & $\begin{array}{l}18,1 \\
15,4\end{array}$ \\
\hline & & & $" t » N . S$. & $u t)=7^{* * *}(1)$ & $u t)=4,7^{* * *}$ \\
\hline \multirow[t]{2}{*}{$29^{\circ} \mathrm{C}$} & \multirow[t]{2}{*}{$\begin{array}{r}108 \\
7\end{array}$} & \multirow[t]{2}{*}{$\begin{array}{l}+ \\
0\end{array}$} & $\begin{array}{l}7,1 \\
6,1 \\
\end{array}$ & $\begin{array}{l}2,9 \\
2,6\end{array}$ & $\begin{array}{l}10 \\
8,7\end{array}$ \\
\hline & & & $\| t n=3,3^{* * *}(1)$ & " $t$ "N.S. & " $t n=3^{* *}$ \\
\hline
\end{tabular}

(1) ** : " $t$ " significatif à $1 \% ; * * *:$ a " significatif à $1 \%$.

(1) ** : « $t$ " signifikant bei $1 \% ; * * *$ : " $t$ " signifikant bei $1 \%$.

4) Influence de l'origine géographique (tableau 3)

L'écotype américain se développe à la même vitesse que l'écotype français soit 18 19 jours à $22^{\circ} \mathrm{C}$ et $10-11$ jours à $29^{\circ} \mathrm{C}$ de l'éclosion à la fin du filage du cocon.

TABL. 3. - Origine géographique de la souche d'abeilles et vitesse $d u$ développement postembryonnaire à $22^{\circ} \mathrm{C}$ et $29^{\circ} \mathrm{C}$.

Tous les individus sont de type monovoltin et sont alimentés avec du Mélilot.

TAB. 3. - Geographische Herkunft der Bienen und postembryonale Entwicklungsgeschwindigkeit bei $22^{\circ} \mathrm{C}$ und $29^{\circ} \mathrm{C}$.

Alle Individuen sind von dem Typ mit einer einzigen Jahresgeneration (monovoltin) und wurden mit Melilotus gefüttert.

\begin{tabular}{|c|c|c|c|c|c|}
\hline \multirow{2}{*}{$\begin{array}{l}\text { Température } \\
\text { Temperatur }\end{array}$} & \multirow{2}{*}{$\begin{array}{c}\text { Nombre } \\
\text { d'individus } \\
\text { Anzahl } \\
\text { der Individuen }\end{array}$} & \multirow{2}{*}{$\begin{array}{c}\text { Origine } \\
\text { de la souche } \\
\text { Herkunft }\end{array}$} & \multicolumn{3}{|c|}{$\begin{array}{l}\text { Durée en jours } \\
\text { Dauer (in Tagen) }\end{array}$} \\
\hline & & & $\begin{array}{c}\text { Stade } \\
\text { d'alimentation } \\
\text { Frassstadium }\end{array}$ & $\begin{array}{c}\text { Filage } \\
\text { du cocon } \\
\text { Spinnen } \\
\text { des Kokons }\end{array}$ & $\begin{array}{c}\text { Développement } \\
\text { larvaire total } \\
\text { Gesamte } \\
\text { Larvenentwicklung }\end{array}$ \\
\hline \multirow[t]{2}{*}{$22^{\circ} \mathrm{C}$} & \multirow[t]{2}{*}{$\begin{array}{l}24 \\
30\end{array}$} & \multirow[t]{2}{*}{$\begin{array}{c}\text { France } \\
\text { Frankreich } \\
\text { Canada } \\
\text { Kanada }\end{array}$} & $\begin{array}{l}12,7 \\
12,7\end{array}$ & $\begin{array}{l}5,5 \\
6\end{array}$ & $\begin{array}{l}18,2 \\
18,6\end{array}$ \\
\hline & & & " $t$ N N.S. & " $t$ » N.S. & "t $»$ N.S. \\
\hline \multirow[t]{2}{*}{$29^{\circ} \mathrm{C}$} & 21 & \multirow[t]{2}{*}{$\begin{array}{c}\text { France } \\
\text { Frankreich } \\
\text { Canada } \\
\text { Kanada }\end{array}$} & 7,6 & 2,9 & 10,2 \\
\hline & & & " $t$ "N.S. & " $t$ "N.S. & "t $"$ N.S. \\
\hline
\end{tabular}


5) Différence de développement entre la première et la deuxième génération annuelle (tableau 4)

A $22^{\circ} \mathrm{C}$ aucune différence n'apparaît entre les individus de première et de deuxième génération. Par contre à $29^{\circ} \mathrm{C}$ le développement des larves pendant la phase d'alimentation est sensiblement plus lent chez les individus de la première génération (8 jours au lieu de 7).

TABL. 4. - Ordre de la génération et vitesse du développement postembryonnaire à $22^{\circ} \mathrm{C}$ et $29^{\circ} \mathrm{C}$ de Megachile pacifica, de souche canadienne.

Tous les individus sont destinés à rentrer en diapause au stade prénymphal; ils se nourrissent sur des provisions de Melilotus alba.

TАВ. 4. - Rangfolge der Generation und postembryonale Entwicklungsgeschwindigkeit der 1. und 2. Generation von Megachile pacifica, Stamm Kanada, bei $22^{\circ} \mathrm{C}$ und $29^{\circ} \mathrm{C}$.

Alle Individuen sind zur Diapause im Praenymphenstadium bestimmt; sie ernähren sich von Vorräten von Melilotus alba.

\begin{tabular}{|c|c|c|c|c|c|}
\hline \multirow[b]{2}{*}{$\begin{array}{l}\text { Température } \\
\text { Temperatur }\end{array}$} & \multirow{2}{*}{$\begin{array}{c}\text { Nombre } \\
\text { d'individus } \\
\text { Anzahl } \\
\text { der Individuen }\end{array}$} & \multirow{2}{*}{$\begin{array}{l}\text { Ordre de la } \\
\text { génération } \\
\text { dans l'année } \\
\text { Rangfolge } \\
\text { der Generation } \\
\text { im Jahr }\end{array}$} & \multicolumn{3}{|c|}{$\begin{array}{l}\text { Durée en jours } \\
\text { Dauer (in Tagen) }\end{array}$} \\
\hline & & & $\begin{array}{c}\text { Stade } \\
\text { d'alimentation } \\
\text { Frassstadium }\end{array}$ & $\begin{array}{c}\text { Filage } \\
\text { du cocon } \\
\text { Spinnen } \\
\text { des Kokons }\end{array}$ & $\begin{array}{c}\text { Développement } \\
\text { larvaire total } \\
\text { Gesamte } \\
\text { Larvenentwicklung }\end{array}$ \\
\hline \multirow[t]{2}{*}{$22^{\circ} \mathrm{C}$} & \multirow[t]{2}{*}{$\begin{array}{l}30 \\
27\end{array}$} & \multirow[t]{2}{*}{$\begin{array}{l}\text { Première } \\
\text { Erste } \\
\text { Deuxième } \\
\text { Zweite }\end{array}$} & $\begin{array}{l}12,7 \\
13,2\end{array}$ & $\begin{array}{l}6 \\
5,3\end{array}$ & $\begin{array}{l}18,7 \\
18,5\end{array}$ \\
\hline & & & " t " N.S. & " $t »$ N.S. & " t $"$ N.S. \\
\hline \multirow[t]{2}{*}{$29^{\circ} \mathrm{C}$} & \multirow[t]{2}{*}{28} & \multirow[t]{2}{*}{$\begin{array}{l}\text { Première } \\
\text { Erste } \\
\text { Deuxième } \\
\text { Zweite }\end{array}$} & 6,6 & 2,9 & $\begin{array}{r}10,6 \\
9,5\end{array}$ \\
\hline & & & ( $t)=4,3^{* *}(1)$ & " $t$ "N.S. & $\| \mathrm{t} n=3,4^{* *}$ \\
\hline
\end{tabular}

(1) ${ }^{* *}$ : « $\mathrm{t}$ » significatif à $1 \%$.

(1) **: a $t$ »signifikant bei $1 \%$.

6) Différence de développement entre mâles et femelles (tableau 5)

La différence de développement est surtout apparente à $22^{\circ} \mathrm{C}$ particulièrement pendant la phase nymphale, les mâles émergeant 7 jours avant les femelles. A $29^{\circ} \mathrm{C}$ cette avance est réduite à 3 jours. 
TABL. 5. - Sexe des individus et développement à $22^{\circ} \mathrm{C}$ et $29^{\circ} \mathrm{C}$ de Megachile pacifica de souche canadienne (Individus bivoltins).

TAв. 5. - Geschlecht der Individuen und Entwicklung bei $22^{\circ} \mathrm{C}$ und $29^{\circ} \mathrm{C}$ von Megachile pacifica, Stamm Kanada. Individuen mit zwei Jahresgenerationen (bivoltin).

\begin{tabular}{|c|c|c|c|c|c|c|c|}
\hline \multirow{3}{*}{$\begin{array}{c}\text { Tempé- } \\
\text { rature } \\
\text { Tempe- } \\
\text { ratur }\end{array}$} & \multirow{3}{*}{$\begin{array}{c}\text { Nombre } \\
\text { d'indi- } \\
\text { vidus } \\
\text { Anzahl } \\
\text { der Indi- } \\
\text { viduen }\end{array}$} & \multirow{3}{*}{$\begin{array}{c}\text { Sexe } \\
\text { Ge- } \\
\text { schlecht }\end{array}$} & \multicolumn{5}{|c|}{$\begin{array}{l}\text { Durée en jours } \\
\text { Dauer (in Tagen) }\end{array}$} \\
\hline & & & $\begin{array}{c}\text { Stade } \\
\text { larvaire }\end{array}$ & $\begin{array}{l}\text { Prénymphe } \\
\text { à nymphe }\end{array}$ & $\begin{array}{l}\text { Nymphe } \\
\text { à imago }\end{array}$ & $\begin{array}{c}\text { Imago } \\
\text { à émergence }\end{array}$ & $\begin{array}{l}\text { Développement } \\
\text { total }\end{array}$ \\
\hline & & & Larve & $\begin{array}{l}\text { Praenymphe } \\
\text { zur Nymphe }\end{array}$ & $\begin{array}{l}\text { Nymphe } \\
\text { zur Imago }\end{array}$ & $\begin{array}{l}\text { Imago bis zum } \\
\text { Ausschlüpfen }\end{array}$ & $\begin{array}{c}\text { Gesamte } \\
\text { Entwicklungszeit }\end{array}$ \\
\hline \multirow[t]{2}{*}{$22^{\circ} \mathrm{C}$} & \multirow[t]{2}{*}{$\begin{array}{l}9 \\
8\end{array}$} & \multirow[t]{2}{*}{$\begin{array}{l}\hat{0} \\
\hat{q}\end{array}$} & $\begin{array}{l}15,4 \\
15,3 \\
\end{array}$ & $\begin{array}{r}9,2 \\
10,3 \\
\end{array}$ & $\begin{array}{l}24,7 \\
28,1\end{array}$ & $\begin{array}{l}5,7 \\
8,8\end{array}$ & $\begin{array}{l}55,0 \\
62,4\end{array}$ \\
\hline & & & $" t » N . S$. & " $t$ $\|=2,2^{*}(1)$ & " $\mathrm{t} n=5,3^{* * *}$ & & $" t \|=3,7^{* *}$ \\
\hline \multirow{2}{*}{$29^{\circ} \mathrm{C}$} & \multirow[t]{2}{*}{$\begin{array}{l}5 \\
2\end{array}$} & \multirow[t]{2}{*}{$\hat{j}$} & $\begin{array}{l}8,8 \\
8,5\end{array}$ & $\begin{array}{l}3,4 \\
3,5\end{array}$ & $\begin{array}{r}8,4 \\
10,0\end{array}$ & $\begin{array}{l}2,8 \\
4,5\end{array}$ & $\begin{array}{l}23,4 \\
26,5\end{array}$ \\
\hline & & & " $t$ "N.S. & " $t$ "N.S. & $u t n=6,5^{* *}$ & & " $\mathrm{t} "=2,5^{*}$ \\
\hline
\end{tabular}

(1) * : " t " significatif à $5 \%$; ** à $1 \%$; *** à $1 \%$.

(1) * : a t " signifikant bei $5 \%$; ** : « t " signifikant bei I \%; *** : " $\mathrm{t}$ " signifikant bei $1 \%$.

\section{B. Délais de réactivation après l'hibernation}

1) Influence des modalités de l'hibernation et de l'incubation sur les délais d'émergence (fig. 3 et 4 )

Les cellules ayant servi à cette expérience sont récoltées après démontage des nichoirs installés dans des champs de luzerne. On a ainsi testé sur des lots de 35 cocons ( 1250 cocons au total ont été utilisés) l'effet de la durée d'hibernation sur les délais d'incubation à $30^{\circ} \mathrm{C}, 22^{\circ} \mathrm{C}$ et en thermopériode : 12 heures à $29^{\circ} \mathrm{C}$ et 12 heures à $15^{\circ} \mathrm{C}$. Les durées d'hibernation varient de 1,5 mois à 10,5 mois à $6^{\circ} \mathrm{C}-7^{\circ} \mathrm{C}$. Plusieurs remarques sont nécessaires après lecture des figures 3 et 4 :

a) Plus la période d'hibernation est longue et plus la période d'incubation nécessaire aux larves pour donner des adultes est courte. Ce phénomène est surtout visible dans le cas d'incubation à $22^{\circ} \mathrm{C}$. Si les larves sont élevées à $30^{\circ} \mathrm{C}$ les délais de réactivation se stabilisent dès que l'hibernation dure plus de 4 mois et demi. Après 9 mois et demi d'hibernation une tendance inverse semble s'instaurer et les délais de l'incubation augmentent.

b) La différence entre les délais d'émergence des lots élevés à $22^{\circ} \mathrm{C}$ et des lots élevés à $30^{\circ} \mathrm{C}$, qui est de 60 jours environ lorsque l'hibernation dure 1 mois et demi s'estompe lorsque l'hibernation s'allonge et se réduit à 25 jours après 8 mois et demi d'hibernation. 


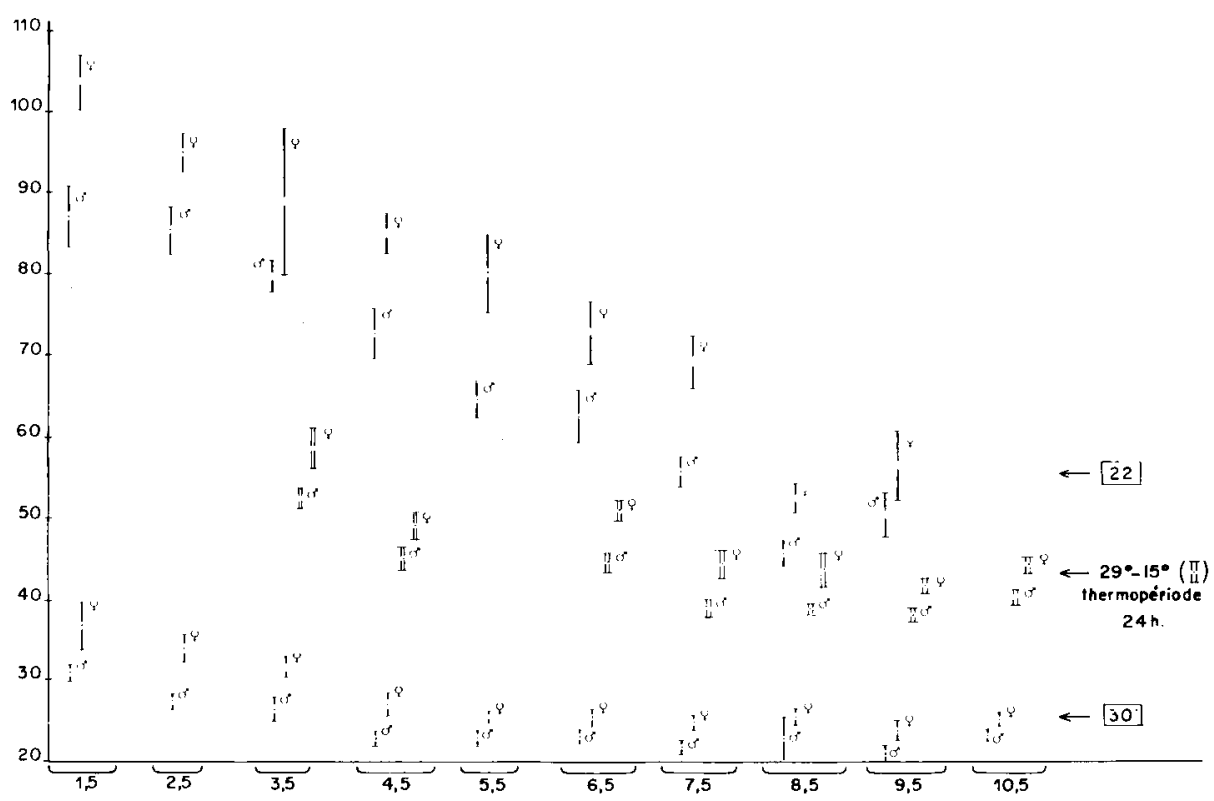

FIG. 3. - Délais d'émergence de mâles et de femelles en fonction de la durée de l'hibernation à $6^{\circ} \mathrm{C}$ et de la température de réactivation.

Verticalement $=$ délais d'émergence en jours.

Horizontalement $=$ durée de l'hibernation en mois.

I $=$ moyenne et son intervalle de confiance $5 \%$.

Aвв. 3. - Verzögerung des Ausschlüpfens der Männchen und Weibchen in Abhängigkeit von der Überwinterungsdauer bei $6^{\circ} \mathrm{C}$ und von der Temperatur der Aktivierung.

Vertikal $=$ Verzögerung des Ausschlüpfens in Tagen.

Horizontal = Uberwinterungsdauer in Monaten.

$I=$ Mittelwert und sein Vertrauensintervall bei $5 \%$.

c) Dans tous les cas les mâles émergent plus tôt que les femelles et la différence entre les délais d'incubation diminue lorsque la durée de l'hibernation augmente. $\mathrm{Ce}$ phénomène est surtout perceptible à $22^{\circ} \mathrm{C}$.

d) L'allongement de l'hibernation réduit la dispersion des émergences dans le temps à partir de 5 mois et demi lorsque la réactivation a lieu à $30^{\circ} \mathrm{C}$. Le phénomène est moins visible à $22^{\circ} \mathrm{C}$. La dispersion des émergences est beaucoup plus prononcée à $22^{\circ} \mathrm{C}$ qu'aux deux autres traitements.

e) L'alternance des températures $29^{\circ} \mathrm{C}$ et $15^{\circ} \mathrm{C}$, autour de la moyenne $22^{\circ} \mathrm{C}$, n'a pas les mêmes conséquences que la température constante $22^{\circ} \mathrm{C}$. Avec le traitement en températures alternées les délais d'émergence sont intermédiaires entre ceux qui sont observés à $22^{\circ} \mathrm{C}$ et ceux qui sont observés à $30^{\circ} \mathrm{C}$. Par ailleurs plus l'hibernation est longue et plus les émergences des lots à $22^{\circ} \mathrm{C}$ et des lots en températures alternées tendent à devenir semblables. Enfin les sorties d'adultes sont beaucoup plus regroupées en températures alternées qu'à température constante quelle que soit la durée de l'hibernation. 

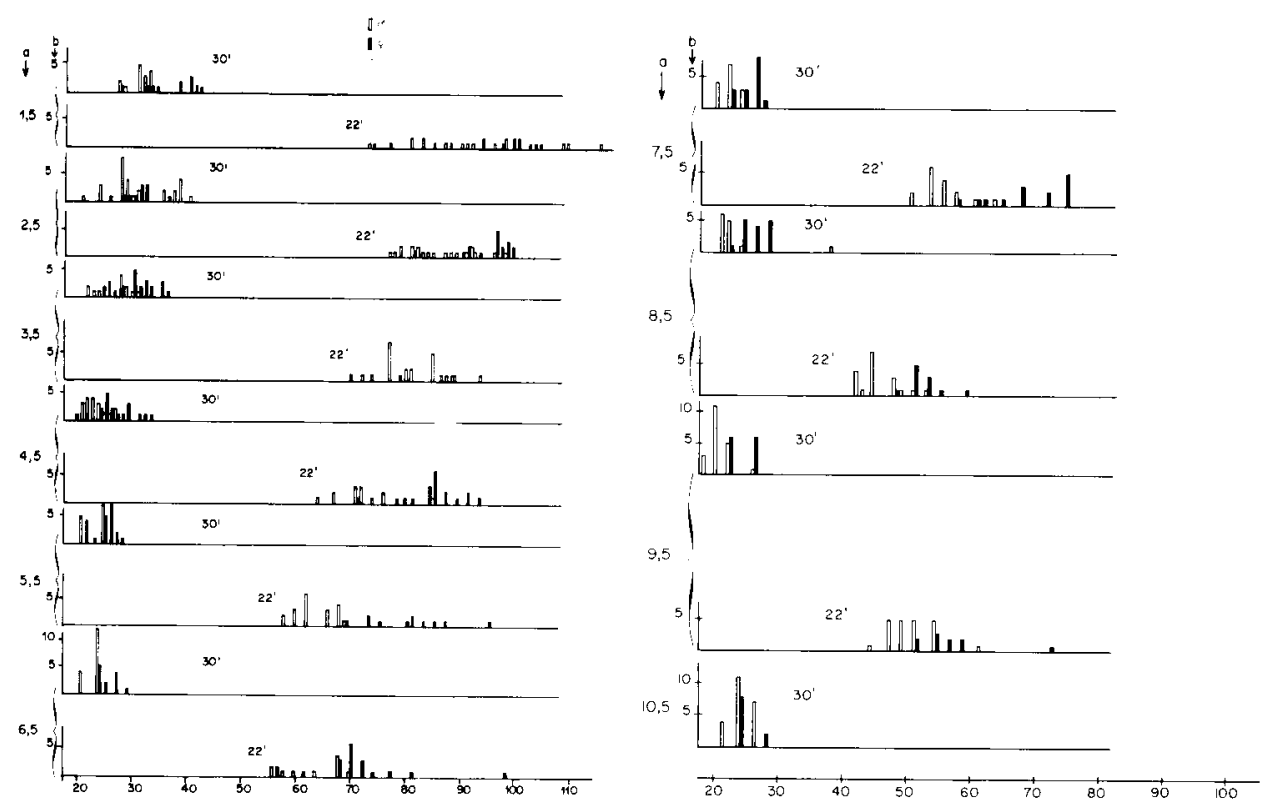

FIG. 4 (A et B). - Émergences de lots d'abeilles ayant subi différentes hibernations et différentes températures de réactivation.

Verticalement $=$ a) Nombre de mois d'hibernation.

b) Fréquence des émergences.

Horizontalement $=$ délais d'émergence avec application de $30^{\circ} \mathrm{C}$ et $22^{\circ} \mathrm{C}$.

Aвв. 4 (A und B). - Ausschlüpfen der Bienengruppen nach unterschiedlicher Überwinterung und unterschiedlichen Aktivierungstemperaturen.

Vertikal =a) Anzahl der Überwinterungsmonate

b) Häufigkeit der schlüpfenden Tiere

Horizontal $=$ Verzögerung des Ausschlüpfens durch Einwirkung einer Temperatur von $30^{\circ} \mathrm{C}$ und $22^{\circ} \mathrm{C}$.
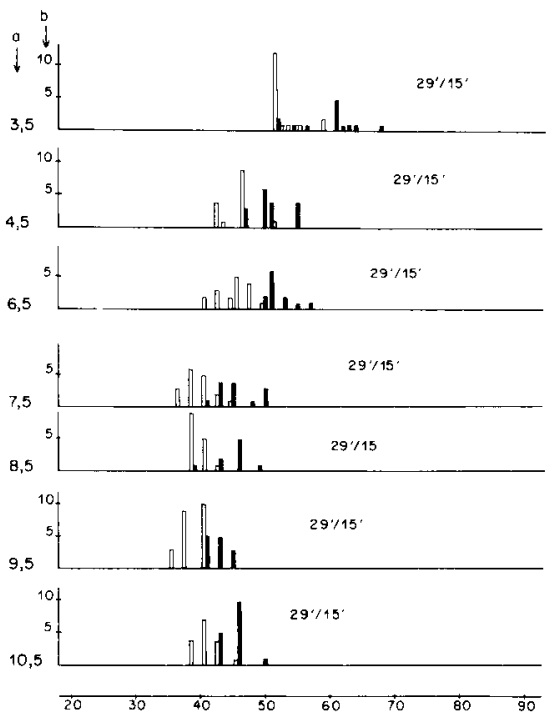

Fig. 4 (C). - même légende que Fig. 4 ( $A$ et $B$ )

Horizontalement : délais d'émergence avec application du régime de températures alternées $29^{\circ} \mathrm{C}$ et $15^{\circ} \mathrm{C}$.

Aвв. 4 (C). - Dieselbe Legende wie für $A b b .4$ ( $A$ und $B$ ).

Horizontal $=$ Verzögerung des Ausschlüpfens durch einen

Temperaturwechsel zwischen $29^{\circ} \mathrm{C}$ und $15^{\circ} \mathrm{C}$. 
2) Influence de l'origine géographique de la souche d'abeilles sur les délais d'émergence (tableau 6)

Deux lots de mégachiles canadiens et français ayant été élevés dans les mêmes conditions on constate que les sorties des individus de souche française des 2 sexes, sont nettement plus tardives que celles des individus de souche américaine.

TABL. 6. - Délais de réactivation à $29^{\circ} \mathrm{C}$ de larves d'origine géographique différente, élevées dans des conditions identiques à partir de l'œuf.

(L'hibernation à 6-7 ${ }^{\circ} \mathrm{C}$ a duré 4 mois.)

TAB. 6. - Aktivierungsverzögerung bei $29^{\circ} \mathrm{C}$ bei Larven verschiedener Herkunft, unter identischen Bedingungen aus dem Ei aufgezogen (Überwinterung bei 6-7 ${ }^{\circ} \mathrm{C}$ durch 4 Monate).

\begin{tabular}{|c|c|c|c|c|}
\hline \multirow{3}{*}{$\begin{array}{l}\text { Origine } \\
\text { de la souche } \\
\text { Herkunft }\end{array}$} & \multicolumn{4}{|c|}{$\begin{array}{l}\text { Délais de réactivation en jours (obtention de la mue imaginale) } \\
\text { Verzögerung der Aktivierung in Tagen (bis zur Imaginalhäutung) }\end{array}$} \\
\hline & \multicolumn{2}{|c|}{$\begin{array}{c}\text { Mâles } \\
\text { Männchen }\end{array}$} & \multicolumn{2}{|c|}{$\begin{array}{l}\text { Femelles } \\
\text { Weibchen }\end{array}$} \\
\hline & $\begin{array}{l}\text { Nombre d'individus } \\
\text { Anzahl der Individuen }\end{array}$ & $\begin{array}{c}\text { Délais } \\
\text { Verzögerung }\end{array}$ & $\begin{array}{l}\text { Nombre d'individus } \\
\text { Anzahl der Individuen }\end{array}$ & $\begin{array}{c}\text { Délais } \\
\text { Verzögerung }\end{array}$ \\
\hline \multirow{2}{*}{$\begin{array}{c}\text { France } \\
\text { Frankreich } \\
\text { Canada } \\
\text { Kanada }\end{array}$} & 9 & $\begin{array}{l}36,9 \pm 2,8 \\
30,5 \pm 1,3\end{array}$ & 3 & $\begin{array}{l}45 \pm 4,3 \\
36,2 \pm 1,1\end{array}$ \\
\hline & & " $t)=3,4^{* *}$ & & $\| t »=5,2^{* * *}$ \\
\hline
\end{tabular}

3) Effet des conditions thermiques de l'élevage des abeilles à partir de la ponte, sur les délais de réactivation après hibernation (tableau 7)

Les 4 lots d'abeilles mentionnés dans le tableau hibernent de la même manière. Les délais d'émergence sont liés aux conditions thermiques de la phase comprise entre la ponte et l'entrée en hibernation. L'application de températures hautes (lots I et II) retarde l'émergence au contraire des températures basses (lots III et IV). Si l'on considère ces deux derniers lots on constate que la seule différence de traitement thermique 
pendant le développement larvaire (application de la température $17^{\circ} \mathrm{C}$, plus longue, pour le lot IV) a des conséquences sensibles sur les délais d'émergence.

TABL. 7. - Effets de conditions d'élevage à partir de l'œuf sur les délais de réactivation à $29^{\circ} \mathrm{C}$, de larves de Megachile pacifica d'origine canadienne.

TAB. 7. - Einfluss der Aufzuchtbedingungen nach dem Schlupf aus dem Ei auf die Aktivierungsverzögerung bei $29^{\circ} \mathrm{C}$, bei Larven von Megachile pacifica, Stamm Kanada.

\begin{tabular}{|c|c|c|c|c|c|}
\hline \multirow{2}{*}{$\begin{array}{c}\quad \mathrm{N}^{0} \text { lot } \\
\text { Nr. der Gruppe }\end{array}$} & \multirow{2}{*}{$\begin{array}{c}\text { Nombre } \\
\text { d'individus } \\
\text { mâles } \\
\text { Anzahl } \\
\text { der Männchen }\end{array}$} & \multicolumn{3}{|c|}{$\begin{array}{l}\text { Conditions d'élevage } \\
\text { Aufzuchtbedingungen }\end{array}$} & \multirow{2}{*}{$\begin{array}{l}\text { Délais } \\
\text { de réactivation } \\
\text { à } 29^{\circ} \mathrm{C} \text { en jours } \\
\text { Verzögerung der } \\
\text { Aktivierung bei } \\
29^{\circ} \mathrm{C} \text { (in Tagen) }\end{array}$} \\
\hline & & $\begin{array}{l}\text { De l'cuf } \\
\text { à la prénymphe } \\
\text { vom Ei } \\
\text { zur Praenymphe }\end{array}$ & $\begin{array}{l}\text { De la prenymphe } \\
\text { à la mise } \\
\text { en hibernation } \\
\text { Von der Praenymphe } \\
\text { zur Einwinterung }\end{array}$ & $\begin{array}{l}\text { Hibernation } \\
\text { Uberwinterung }\end{array}$ & \\
\hline I & 14 & $29^{\circ} \mathrm{C}$ & $\begin{array}{l}3 \text { semaines à } 29^{\circ} \mathrm{C} \\
3 \text { Wochen bei } 29^{\circ} \mathrm{C}\end{array}$ & & $37 \pm 4,1$ \\
\hline II & 39 & $22^{\circ} \mathrm{C}$ & $\begin{array}{l}3 \text { semaines à } 22^{\circ} \mathrm{C} \\
3 \text { Wochen bei } 22^{\circ} \mathrm{C}\end{array}$ & & $31 \pm 0,9$ \\
\hline III & 19 & $\begin{array}{l}15 \text { jours à } 22^{\circ} \mathrm{C} \\
\text { puis } 17^{\circ} \mathrm{C} \\
15 \text { Tage bei } 22^{\circ} \mathrm{C} \\
\text { dann } 177^{\circ} \mathrm{C}\end{array}$ & $\begin{array}{l}6 \text { semaines à } 17^{\circ} \mathrm{C} \\
3 \text { Wochen bei } 17^{\circ} \mathrm{C}\end{array}$ & $\left\{\begin{array}{l}4 \text { mois à } 6^{\circ} \mathrm{C} \\
4 \text { Monate bei } 6^{\circ} \mathrm{C}\end{array}\right.$ & $27 \pm 1,2$ \\
\hline IV & 26 & $\begin{array}{l}5 \text { jours à } 22^{\circ} \mathrm{C} \\
\text { puis } 17^{\circ} \mathrm{C} \\
5 \text { Tage bei } 22^{\circ} \mathrm{C} \\
\text { dann } 17^{\circ} \mathrm{C}\end{array}$ & $\begin{array}{l}6 \text { semaines à } 17^{\circ} \mathrm{C} \\
3 \text { Wochen bei } 17^{\circ} \mathrm{C}\end{array}$ & & $23 \pm 1,3$ \\
\hline
\end{tabular}

4) Influence de la date de ponte sur les délais de réactivation (tableau 8)

Les larves issues d'un élevage en champ et contenues dans des nichoirs semblables sont séparées en deux lots : celui qui provient d'une ponte du 15 juillet et celui qui provient d'une ponte tardive un mois plus tard. Après une hibernation et une incubation communes on observe un net retard de reprise du développement chez les larves du deuxième lot.

5) Influence du bois du nichoir sur les délais de réactivation (tableau 9)

Les larves de souche canadienne s'étant développées dans les conditions extérieures, soit dans des nichoirs en Pin, soit dans des nichoirs en Sipo, hivernent dans les mêmes conditions. On constate que les larves mâles et femelles élevées dans le Pin se nymphosent nettement plus tard que celles qui sont issues des nichoirs en Sipo. 
TABL. 8. - Influence de la date de ponte sur les délais de réactivation après 4 mois d'hibernation d̀ $6^{\circ} \mathrm{C}$, de larves de souche canadienne élevées en champ dans des nichoirs de bois $d$ 'Entandrophragma (Sipo).

TAB. 8. - Einfluss des Legedatums auf die Aktivierungsverzögerung nach \& Monaten Überwinterung bei $6^{\circ} \mathrm{C}$ bei Larven vom Stamm Kanada, aufgezogen im Freien in Nisthöhlen im Holz von Entandrophragma (Sipo).

\begin{tabular}{|c|c|c|c|c|c|c|c|}
\hline \multirow{2}{*}{$\begin{array}{l}\text { Période } \\
\text { de ponte } \\
\text { Periode } \\
\text { der } \\
\text { Eiablage }\end{array}$} & \multirow{2}{*}{$\begin{array}{c}\text { Effectifs } \\
\text { Anzahl }\end{array}$} & \multicolumn{6}{|c|}{$\begin{array}{c}\text { Délais de réactivation à } 22^{\circ} \mathrm{C} \text { en jours } \\
\text { Verzögerung der Aktivierung (in Tagen) }\end{array}$} \\
\hline & & \multicolumn{2}{|c|}{$\begin{array}{l}\text { De la prénymphe } \\
\text { à la nymphe } \\
\text { Von der Praenymphe } \\
\text { zur Nymphe }\end{array}$} & \multicolumn{2}{|c|}{$\begin{array}{l}\text { De la nymphe } \\
\text { à l'imago } \\
\text { Von der Nymphe } \\
\text { zur Imago }\end{array}$} & \multicolumn{2}{|c|}{$\begin{array}{l}\text { Développement } \\
\text { total } \\
\text { Gesamtentwicklung }\end{array}$} \\
\hline \multirow{4}{*}{$\begin{array}{l}15 \text { juillet } \\
15 \text { Juli } \\
15 \text { août } \\
15 \text { August }\end{array}$} & \multirow{4}{*}{$\begin{array}{l}180 \\
\text { et } 59 \\
140 \\
\text { et } 99\end{array}$} & $\hat{0}$ & q & $\hat{o}$ & q & $\hat{j}$ & q \\
\hline & & 27,9 & 38,6 & 26,4 & 26,2 & 53,8 & 64,8 \\
\hline & & 39,8 & 50,1 & 25,4 & 25,2 & 65,1 & 75,3 \\
\hline & & at $t=3,1^{* *}$ & a $t$ "N.S. & *t $\#$ N.S. & " $t » N . S$. & * $t »=3,0^{* *}$ & $\alpha t *=2,1^{*}$ \\
\hline
\end{tabular}

TABL. 9. - Influence $d u$ bois du nichoir sur les délais de réactivation de larves issues de la souche canadienne, de la même période de ponte et ayant hiberné 8 mois à $6^{\circ} \mathrm{C}$.

ТАВ. 9. - Einfluss des Holzes der Nisthöhle auf die Verzögerung der Aktivierung bei Larven vom Stamm Kanada, die aus derselben Eilegeperiode kamen und 8 Monate lang bei $6^{\circ} \mathrm{C}$ überwintert hatten.

\begin{tabular}{|c|c|c|c|c|}
\hline \multirow{3}{*}{$\begin{array}{l}\text { Bois du nichoir } \\
\text { Holz der Nisthöhle }\end{array}$} & \multicolumn{4}{|c|}{$\begin{array}{c}\text { Délais de réactivation à } 22^{\circ} \mathrm{C} \text { en jours (Obtention de la mue imaginale) } \\
\text { Verzögerung der Aktivierung bei } 22^{\circ} \mathrm{C} \text { (in Tagen) }\end{array}$} \\
\hline & \multicolumn{2}{|c|}{$\begin{array}{l}\text { Mâles } \\
\text { Männchen }\end{array}$} & \multicolumn{2}{|c|}{$\begin{array}{l}\text { Femelles } \\
\text { Weibchen }\end{array}$} \\
\hline & $\begin{array}{c}\text { Nombre d'individus } \\
\text { Zahl der Individuen }\end{array}$ & $\begin{array}{c}\text { Délais } \\
\text { Verzögerung }\end{array}$ & $\begin{array}{l}\text { Nombre d'individus } \\
\text { Zahl der Individuen }\end{array}$ & $\begin{array}{c}\text { Délais } \\
\text { Verzögerung }\end{array}$ \\
\hline \multirow[t]{2}{*}{$\begin{array}{l}\text { Pinus } \\
\text { Entandrophragma } \\
\text { (Sipo) }\end{array}$} & 17 & 19,9 & $\begin{array}{l}7 \\
8\end{array}$ & $\begin{array}{l}29,3 \\
21,9\end{array}$ \\
\hline & & $u t \|=2,1^{*}$ & & " $t n=3,1^{* *}$ \\
\hline
\end{tabular}




\section{DISCUSSION}

\section{Sur les facteurs du développement pendant la période estivale}

Selon Bohart (non publié) la température idéale pour le développement de Megachile pacifica se situe entre 28 et $31^{\circ} \mathrm{C}$. Avec ce traitement thermique la durée du développement est de 11 à 13 jours ce qui correspond à nos résultats. Lorsque le pollen de luzerne fait partie de la nourriture, on assiste à un développement plus rapide. Selon LEVIN et HAYDAK (1957) travaillant sur Osmia lignaria, la valeur nutritive d'un pollen est mesurée grâce à l'estimation de la durée des stades larvaires s'alimentant, et du poids des larves. Par cette méthode simple on pourrait tester d'autres pollens sur $M$. pacifica, afin de connaitre le plus nutritif.

Le ralentissement du développement chez les larves qui vont entrer en diapause a déjà été signalé chez Cydia par Siegler et Simanton (1915), chez Laspeyresia par Dickson (1949), chez Telea par Dawson's (1931) cités par ANDrEWARTHA (1952). Le même phénomène est observé chez Osmia coerulescens (TASEI, 1972). Nos résultats prouvent que la diapause est induite à un stade très précoce, dès la ponte et peut-être même avant la mue imaginale de la mère si l'on se réfère au travail de BITNER (1976). L'entrée en diapause ne peut être arrêtée par un quelconque refroidissement en fin de développement larvaire, puisqu'à $15^{\circ} \mathrm{C}$ les larves peuvent se nymphoser. Ceci est confirmé par les dires de plusieurs chercheurs, particulièrement MANNINGER et TORCHIO (Communications personnelles). Ce résultat est opposé aux conceptions de StEPHEN (1962) qui pense que la deuxième génération est induite par la somme des températures subies par la larve.

\section{Sur les facteurs influant sur les délais de développement à la sortie de l'hibernation}

L'influence de la longueur de l'hibernation sur les délais de réactivation est bien connue d'une manière générale chez les insectes en diapause et chez $M$. pacifica en particulier (KRUNiC et HinKs, 1972; Johansen et Eves, 1973). Nos résultats font ressortir certaines particularités :

- La dispersion des émergences à $30^{\circ} \mathrm{C}$ se réduit après 5 mois d'hibernation et après 9 mois si la température de réactivation égale $22^{\circ} \mathrm{C}$.

- Les délais d'émergence sont d'autant plus réduits que l'hibernation est longue. Cependant à partir d'une certaine longueur d'hibernation : 8 à 10 mois, les délais augmentent. Une telle régression de l'état physiologique est signalée chez Psylla buxi par NgUyen (1968). Chez $M$. pacifica une prolongation de l'hibernation au-delà de 20 mois à $4-5^{\circ} \mathrm{C}$, induit une mortalité très élevée : $75 \%$ selon CiURDAREsCu (1974) ou totale (JoHANSEN et Eves, 1973; TASEI, non publié) ce qui parait enlever tout espoir de conserver au froid les cocons durant 2 années consécutives. 
L'étude de l'influence de la température de réactivation nous conduit à deux constatations :

- La température de $30^{\circ} \mathrm{C}$ est certainement la plus favorable au développement des individus sortant de 4 à 5 mois d'hibernation à $5^{\circ} \mathrm{C}$. Ce résultat est en accord avec ceux de CiURdaresCU (1974) et avec toutes les recommandations faites aux éleveurs de mégachiles, en particulier celles de Новвs (1973).

- L'application de températures alternées n'augmente pas la proportion d'individus qui restent en diapause prolongée après l'hibernation comme l'écrivent STEPHEN et OsGOod (1965). Au contraire l'effet de l'alternance $29^{\circ} \mathrm{C}-15^{\circ} \mathrm{C}$ parait bénéfique : c'est ainsi que les délais sont raccourcis par rapport à ceux qui correspondent à la température moyenne constante de $22^{\circ} \mathrm{C}$ et la mortalité dans les lots en températures alternées est inférieure à celle qui est observée dans les lots en température constante $(3,6 \%$ à $29^{\circ} \mathrm{C}-15^{\circ} \mathrm{C}$ et $15,2 \%$ à $22^{\circ} \mathrm{C}$ ).

L'influence des conditions d'élevage avant l'entrée en hibernation sur l'intensité de la diapause n'est pas un sujet abordé par les auteurs. Seuls JoHANSEN et Eves (1973) et KRUNIC et HINKs (1972) étudient l'effet de températures intermédiaires "préparant " l'hibernation, sur la mortalité et sur la levée de la diapause pendant l'application des températures basses. Nous constatons que les œufs pondus précocement en saison donnent des larves qui ont une diapause moins intense que ceux qui sont pondus un mois plus tard. Il est probable que dans la nature une préparation à l'hibernation (températures nocturnes estivales par exemple) a rendu cette dernière plus efficace et a permis une élimination de la diapause plus rapide. On rejoint ici les conclusions de KRUNIC et HiNKs et l'on peut trouver dans ce phénomène une explication aux différences d'émergence en laboratoire entre individus d'origine méridionale issus de ponte précoce, et individus d'origine septentrionale issus de ponte tardive (TASEI, 1973). En conditions contrôlées l'application de la température $17^{\circ} \mathrm{C}$ pendant le développement larvaire, réduit l'intensité de la diapause, ce qui montre que les larves à diapause peuvent arriver à l'entrée de l'hibernation dans des états physiologiques différents.

La souche monovoltine française a une diapause plus intense que la souche polyvoltine américaine. Ces deux souches sont pourtant identiques lorsque l'on compare la rapidité du développement larvaire après la ponte.

\section{CONCLUSIONS}

1. Les larves de M. pacifica se développent avec le plus de rapidité et de synchronisme à une température proche de $30^{\circ} \mathrm{C}$. La qualité de la nourriture influe sur les délais de développement : l'association du pollen de Melilot et du pollen de Luzerne est plus favorable que le seul pollen de Melilot.

2. Les larves qui vont entrer en diapause ont un développement ralenti. 
3. Un traitement à basse température $\left(15^{\circ} \mathrm{C}\right)$ en fin de développement larvaire ne peut empêcher les larves destinées à se nymphoser, de poursuivre leur développement.

4. Un traitement à basse température $\left(17^{\circ} \mathrm{C}\right)$ appliqué pendant la période d'alimentation à des larves destinées à entrer en diapause réduit l'intensité de cette diapause.

5. La longueur de l'hibernation et la température de réactivation sont les principaux facteurs déterminant la durée de la réactivation. On assiste à une régression de l'état physiologique lorsque l'on porte la durée de l'hibernation à $5^{\circ} \mathrm{C}-7^{\circ} \mathrm{C}$, au-delà de $8-10$ mois.

6. Les températures alternées semblent mieux convenir à la réactivation, que les températures constantes.

7. La souche monovoltine française se distingue de la souche polyvoltine américaine par une intensité de diapause sensiblement supérieure. Les inconvénients du bivoltinisme de la souche américaine en France conduisent à mieux connaitre la souche monovoltine et à la multiplier.

8. La pluralité des facteurs influant sur le développement doit engager les chercheurs à la prudence et à la rigueur méthodologique dans le cours des études sur la physiologie des Apoïdes.

Reçu pour publication en février 1978. Eingegangen im Februar 1978.

\section{ZUSAMMENFASSUNG}

\section{Einleitung}

Während die Aufzuchttechnik der Luzerne-Blattschneiderbienen Gegenstand zahlreicher Untersuchungen war, hat man sich mit ihrer Entwicklungsphysiologie bisher nur wenig beschäftigt. Unsere Arbeit analysiert einige Ursachen der Variation der Entwicklungsgeschwindigkeit in verschiedenen Abschnitten des Zyklus.

\section{Material und Methode}

Abb. 1 gibt einen Úberblick über die zeitliche Einordnung und die Charakterisierung der verschiedenen Perioden des Jahreszyklus. Unter kontrollierten Bedingungen wurde die Larvenentwicklung durch individuelle Aufzuchten sovohl in der Sommerphase wie in der Phase nach der Uberwinterung beobachtet. Diese Methode ermöglichte einen Vergleich der Entwicklung der Biene nach ihrer geographischen Herkunft, dem Zustand der Diapause, der Rangordnung der Generation (1. oder 2. Flug), der Temperaturbehandlung, der Ernährung der Larven usw.

\section{Die wichtigsten Resultate}

1. Bei einer konstanten Temperatur von $15^{\circ} \mathrm{C}$ sterben die Eier und jungen Larven $\mathrm{ab}$, nur die älteren Larven entwickeln sich weiter und gelangen zur Verpuppung. Bei $17^{\circ} \mathrm{C}$ überschreitet die Mortalität $20 \%$ der Individuen. Die Entwicklungsdauer beträgt 47 Tage (vom Ei bis zum Spinnen des Kokons). Bei $22^{\circ} \mathrm{C}$ ist diese Entwicklungszeit auf 24 Tage und bei $29^{\circ} \mathrm{C}$ auf 13 Tage verkürzt (Abb. 2).

2. Eine Mischung des Pollens von Melilotus und von Medicago sativa (Luzerne) fördert die Entwicklung stärker als Pollen von Melilotus allein (Tab. 1). 
3. Die Larven, die zur Diapause bestimmt sind, entwickeln sich langsamer als diejenigen, die sich später verpuppen werden, um eine zweite Generation zu ergeben (Tab. 2). Diese Feststellung ergab sich auch bei anderen Insekten (ANDREWARTHA, 1952).

4. Tiere des ersten Fluges ergeben eine Nachkommenschaft mit langsamerer Entwicklung als die Nachkommen des zweiten Fluges (Tab. 4).

5. Je länger die Überwinterungsphase, desto kürzer die Inkubation. Dieses Phänomen zeigt sich vor allem bei $22^{\circ} \mathrm{C}$. Wenn die Larven bei $30^{\circ} \mathrm{C}$ aufgezogen werden, stabilisiert sich die Verzögerung der Aktivierung, sofern die Uberwinterung mehr als 4 1/2 Monate gedauert hat. Nach einer Winterruhe von $91 / 2$ Monaten stellt sich eine umgekehrte Tendenz ein und die Verzögerung der Aktivierung nimmt zu. Ähnliche Resultate wurden bei Psylla buxi erzielt (NGUYEN, 1968).

Die Verlängerung der Winterruhe verringert die Streuung der Schlüpftermine. Ein Wechseln zwischen den Temperaturen $29^{\circ} \mathrm{C}$ und $15^{\circ} \mathrm{C}$ (Mittel $22^{\circ} \mathrm{C}$ ) führt zu einer Abkürzung der Verzögerung des Ausschlüpfens und zu einer Verringerung sowohl der zeitlichen Streuung des Ausschlüpfens wie der Larvenmortalität, im Vergleich zu einer konstanten Temperatur von $22^{\circ} \mathrm{C}$ (Abb. 3 u. 4).

6. Der französische Stamm der Megachile benötigt zum Ausschlüpfen eine längere Inkubation als der kanadische (Tab.6). In der Sommerperiode zeigen aber beide Stämme die gleiche Entwicklungsgeschwindigkeit (Tab. 3).

7. Die Verzögerungen beim Schlüpfen hängen von den Temperaturverhältnissen zwischen der Ablage der Eier und dem Beginn der Einwinterung ab. Hohe Temperaturen bei der Aufzucht verzögern das Ausschlüpfen, während niedrige Temperaturen den gegenteiligen Effekt haben - selbst wenn diese nur während der Frassperiode der Larve eingewirkt haben (Tab. 7).

8. Larven, die aus einem zeitigen Gelege (1. Juli) stammen, erreichen den Winterbeginn mit einem physiologischen Vorsprung : Bei ihnen wird die Diapause leichter beendet als bei solchen, die aus einem späten Gelege (1. August) stammen (Tab. 8).

\section{BIBLIOGR APHIE}

ANDrewartha H. G., 1952. - Diapause in relation to the Ecology of insects. Biol. Rev., 27, 50-107.

BITNER R. M., 1976. - Ecological management of the alfalfa leaf-cutter bee Megachile pacifica Panz. with emphasis on diapause induction. $\mathrm{Ph} . \mathrm{D}$. Thesis, Utah State Univ. Logan.

Bohart G. E. - non publié.

Ciurdarescu G., 1974. - The effects of storage length and incubation temperatures on the development of Megachile rotundata F. larvae. Probl. Prot. Plant, 2, 225-230.

Hosbs G. A., 1973. - Alfalfa leafcutter bees for pollinating alfalfa in western Canada. Pub. 1945. Information division Canad. Dep. Agric.

JohANSEN C. A., Eves J. D., 1973. - Effects of chilling, humidity and seasonal conditions on emergence of the alfalfa leafcutting bee. Environ. Entomol., 2, 23-26.

KRUNIC M. D., HINKs C. F., 1972. - The effect of temperature and temperature pretreatment on diapause and on the synchronization of adult emergence in Megachile rotundata (Hym. megachilidae). Canad. Entomol., 104, 889-893.

LEVIN M. D., HAYDAK M. H., 1957. - Comparative value of different pollens in the nutrition of Osmia lignaria, Bee Wld., 38, 221-226.

MANNINgeR S. - Communication personnelle.

NguYen T. X., 1968. - Rôle de la température dans l'évolution et l'élimination de la diapause larvaire de Psylla buxi (Hom. psyllidae) Ann. Soc. Entomol. Fr., 4, 69-74. 
StePHEN W. P., 1962. - Propagation of the leaf-cutter bee for alfalfa seed production. Stn. Bull. 586. Agr. Exp. Stn. Oregon St Univ., 16 p.

STEPHEN W.P., Osgood C.E., 1965. - The induction of emergence in the leaf-cutter bee Megachile rotundata an important pollinator of alfalfa. J. Econ. Entomol., 58, 284-286.

TASEI J. N., 1972. - Observations préliminaires sur la biologie d'Osmia (Chalcosmia) coerulescens L. (Hymenoptera megachilidae) pollinisatrice de la luzerne (Medicago sativa L.) Apidologie, 3, 149-165.

TASEI J. N., 1973. - Observations sur le développement d'Osmia cornuta Latr. et Osmia rufa L. (Hym. Megachilidae). Apidologie, 4, 295-315.

Torchio P. F. - Communication personnelle. 